\title{
Damped Oscillations - A smartphone approach
}

\author{
M. Santamaría, E. S. Cruz de Gracia, L. S. Dorneles
}

\begin{abstract}
The study of the influence of geometric factors on an oscillating pendulum under various damping conditions is reported. Different cross-section areas perpendicular to the motion of the pendulum mass were studied. A smartphone was used as a pendulum and at the same time as a data recorder. Results show that the smartphone is an effective and reliable tool to be used when performing educational activities, and at the same time it presents students with a variety of ways for learning new content and physical concepts. It offers the opportunity to carry out experiments in the classroom, in the laboratory, or at home. In this way, the increment in the crosssection area slightly increases the damping coefficient, and rapidly decreases the oscillation amplitude as time passes. Additionally, the time necessary to decrease the amplitude by half is inversely proportional to the cross-section area of the pendulum. As expected, no significant variation in the period nor the angular frequency were found, due to the air-pendulum drag properties and to the slow pendulum speed.
\end{abstract}

Index Terms - Cross-Section Area, damped Oscillations, damping coefficient, half-life of the amplitude, pendulum mass, smartphone.

\section{INTRODUCTION}

Smartphones have several sensors that allow one to record changes in the state of their position through movements such as rotation, tilt, or linear acceleration; one is also capable to register environmental changes in his/her immediate surroundings, including the amount of light, sound intensity, and atmospheric pressure [1]. This opens the possibility to carry out academic and scientific experimentation activities in the classroom, in the laboratory, and at home.

Usually, we find two different types of sensors in smartphones. The first type, physical sensors, are miniaturized solid-state devices that process mechanical and electrical signals (MEMS) [2]. Those based on hardware, such as accelerometer, gyroscope, and magnetometer, belong to this first type. In the second type, derived sensors, operation depends on both hardware and software. The rotation vector, the linear acceleration, and the step detector belong to this second type. Depending on the operating system, there are free applications that allow managing each type of sensor and resource available to perform academic activities as a complement to the theoretical classes. Sensor Box [3], Sensor Kinetics [4], Phyphox [5], Science Journal [6] are some of these applications.

In this way, a technological tool is available to most students, which allows for the teacher to use a novel way to address many contents regarding physical experiments. Also, it enables the demonstration of some physical-mathematical models that describe natural phenomena. The academic activities proposed by Vogt and Kuhn (2012) use the smartphone's accelerometer to measure the acceleration of bodies in free fall in addition to studying the movement for a mathematical pendulum [7] and [8]. Shakur and Kraft (2016) use the gyroscope and the accelerometer to measure the Coriolis acceleration [9]. Groff (2019) uses the sound level meter to measure an RC circuit time constant [10].

The mathematical pendulum is a physical system made up of a body of mass $m$ suspended at the end of a very light and inextensible thread of length $l[11]$. Fig. 1a shows a diagram of this physical system, which upon being laterally displaced from its vertical equilibrium position by a small initial angle $\theta_{o}$ and released, will describe an arc of circumference $s_{o}$ along its trajectory. Under vacuum, without the effect of air resistance, the system would oscillate indefinitely with a constant amplitude. However, in real experiments, the environment influences the pendulum movement leading to losses in its mechanical energy due to air resistance. Consequently, a continuous decrease in the amplitude is observed [12].

\footnotetext{
M. Santamaría, Universidad de Panamá, Departamento de Física. Centro de Investigaciones con Técnicas Nucleares (CITEN), Panamá (marciano.santamaria@up.ac.pa).

E. S. Cruz de Gracia, Universidad Tecnológica de Panamá, Facultad de Ingeniería Eléctrica, Panamá (evgeni.cruz@utp.ac.pa)

L. S. Dorneles, Universidade Federal de Santa Maria, Departamento de Física, Av. Roraima, 1000, Camobi, Santa Maria, RS, Brazil, 97105-900 (lucio.dorneles@ufsm.br) 


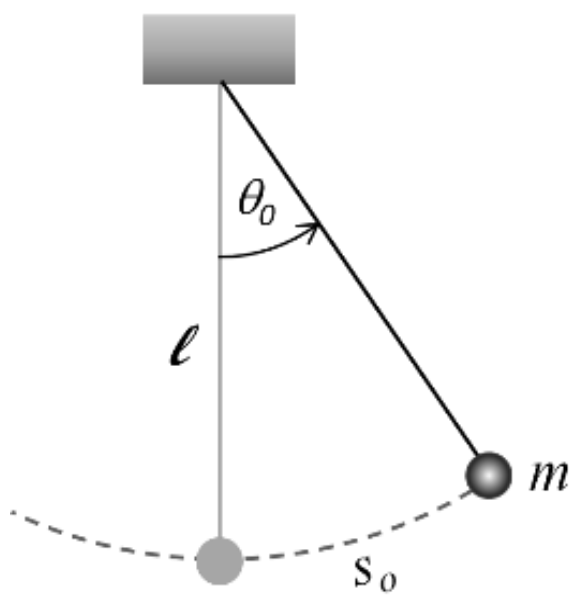

(a)

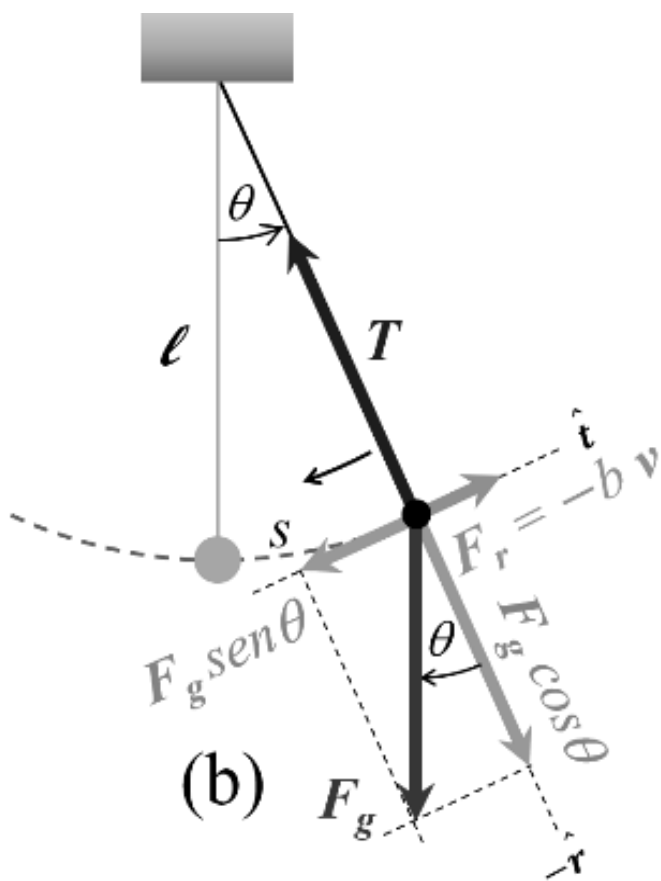

Fig. 1. (a) Diagram of a pendulum of length $l$ and mass $m$ laterally displaced by an angle $\theta_{o}$ from its equilibrium position. (b) Diagram of the forces acting on the pendulum mass in a specific point of the trajectory; $\hat{t}$ is the direction tangential to the path and $\hat{r}$, the radial direction.

The dynamic analysis of this movement based on the Newtonian formalism for a specific point of the trajectory, as indicated in fig. $1 \mathrm{~b}$, shows the existence of radial and tangential forces acting on the body. Radial to the arc of circumference described and oriented towards the center, the tension force of the thread (T) acts at all times. Similarly, the gravitational force (weight) perpendicular to the path and oriented out of the arc of circumference acts at all times. Radial forces are unbalanced, except at the extremes of motion, and are responsible for centripetal acceleration experienced by the body. The equation of motion of the system, perpendicular to the trajectory, obtained from Newton second law is,

where $\boldsymbol{a}_{\boldsymbol{r}}$ is the radial acceleration, and its magnitude is given by,

$$
\boldsymbol{T}-m \boldsymbol{g} \cos \theta=m \boldsymbol{a}_{\boldsymbol{r}}
$$

$$
a_{r}=\frac{v_{t}^{2}}{r}
$$

where $v_{t}$ is the modulus of the tangential velocity at the particular instant described in figure 1.b. It is clear from equations (1) and (2) that at the extreme points of the motion, when the pendulum mass remains momentarily at rest $\left(v_{t}=0\right)$, the radial acceleration at those instants is zero and, the radial forces will be momentarily balanced.

Fig. $1 \mathrm{~b}$ shows the component of the gravitational force parallel to the trajectory and the dissipative force due to air resistance. The component of the gravitational force is oriented towards the system equilibrium position $(\theta=0)$, it is a restoring force. This behavior is independent of the direction of motion of the body [13]. In turn, the dissipative force is proportional to the modulus of the body velocity, and is oriented in the opposite direction [14]. The equation of motion of the system, parallel to the trajectory, obtained from Newton second law is,

where $b$ is the damping constant expressed in $\mathrm{kg} / \mathrm{s}$, and $\boldsymbol{a}_{\boldsymbol{t}}$ is the tangential acceleration.

$$
-m \boldsymbol{g} \operatorname{sen} \theta-b \boldsymbol{v}_{t}=m \boldsymbol{a}_{t}
$$

In terms of the angular position $\theta$, using $v_{t}=d s / d t, a_{t}=d^{2} s / d t^{2}$ and $s=l \theta$, with $\theta$ in radians, equation (3) becomes,

$$
-m g \operatorname{sen} \theta-b l \frac{d \theta}{d t}=m l \frac{d^{2} \theta}{d t^{2}} \text {. }
$$

It is possible to approximate $\operatorname{sen} \theta \cong \theta$ for small oscillation angles $\left(\theta<15^{\circ}\right)$, and rewrite equation (4) for $\theta$ expressed in radians,

$$
\frac{d^{2} \theta}{d t^{2}}+\frac{b}{m} \frac{d \theta}{d t}+\frac{g}{l} \theta=0 .
$$

The solution of the differential equation (5) allows determining the time-dependent pendulum angular position,

$$
\theta(t)=\theta_{o} e^{-\gamma t} \operatorname{sen}\left(w^{\prime} t+\varphi\right) \text {. }
$$

Thus, $\theta(t)$ represents the angular position of the pendulum at any instant of time $t, \theta_{o}$ is the initial angular displacement at $t=0 s, \gamma(=b / 2 m)$ is the damping coefficient of the system measured in $s^{-1}, \varphi$ is the initial phase constant of the motion and $w^{\prime}$ is the angular frequency, given by equation (7) when dissipative force is present in the system,

$$
w^{\prime}=\sqrt{\frac{g}{l}-\gamma^{2}}=\sqrt{\frac{g}{l}-\left(\frac{b}{2 m}\right)^{2}} .
$$


Therefore, in equation (6) the term $\theta_{o} e^{-\gamma t}$, represents a time-dependent (decreasing) amplitude of oscillation due to dissipative forces, and the term $\operatorname{sen}\left(w^{\prime} t+\varphi\right)$ represents the periodic behavior of the motion. Furthermore, the second derivative gives the tangential acceleration of the oscillating mass. Finally, it is also possible to study the damping coefficient effect on the acceleration because of the exponential term immutability with derivative order.

Using equation (7), it is possible to predict that for $\gamma<1, w^{\prime}$ will not be significantly changed from the expected value of 2,9 $\mathrm{rad} / \mathrm{s}$. For $g / l=8,4 \mathrm{~s}^{-2}$, and using the values measured in this article, $\gamma<0,08 \mathrm{~s}^{-1}$, the contribution from $\gamma^{2}$ to $g / l$ would be $<$ $0,007 s^{-2}$.

\section{METHODOLOGY}

The acceleration sensor of a smartphone was used to investigate the behavior of the radial and tangential acceleration experienced by the device working as the body of an oscillating pendulum.

The linear acceleration sensor of a Samsung Galaxy SM-A750G smartphone with an Android V9.0 operating system (168,0 g mass, with dimensions $76,8 \mathrm{~mm} \times 159,8 \mathrm{~mm}$ ) [15], was used to determine the tangential and radial acceleration experienced by a pendulum oscillating in a vertical plane. The pendulum body, made up of the telephone itself, was fixed with two ropes to the frame of the laboratory door.

The length of the pendulum was $116,5 \mathrm{~cm}$, and the pendulum body was laterally displaced from its equilibrium position by an angle of less than $15^{\circ}$ and subsequently released. An additional thread was tied at the geometrical center of the body to reduce swaying, so the tension line of action of this additional thread would pass through the device's center of gravity.

Four different tests were carried out to study the environmental influence on the pendulum oscillation. In each test a light cardboard barrier was fixed to the back of the device. Cardboard was used to increase cross-section area without significantly changing the pendulum mass. Fig. 2 shows the experimental setup. For each test a different cross-section area was used, 122,7 $\mathrm{cm}^{2}$ (smartphone without cardboard barrier), $300,4 \mathrm{~cm}^{2}, 450,0 \mathrm{~cm}^{2}$, and $603,2 \mathrm{~cm}^{2}$.

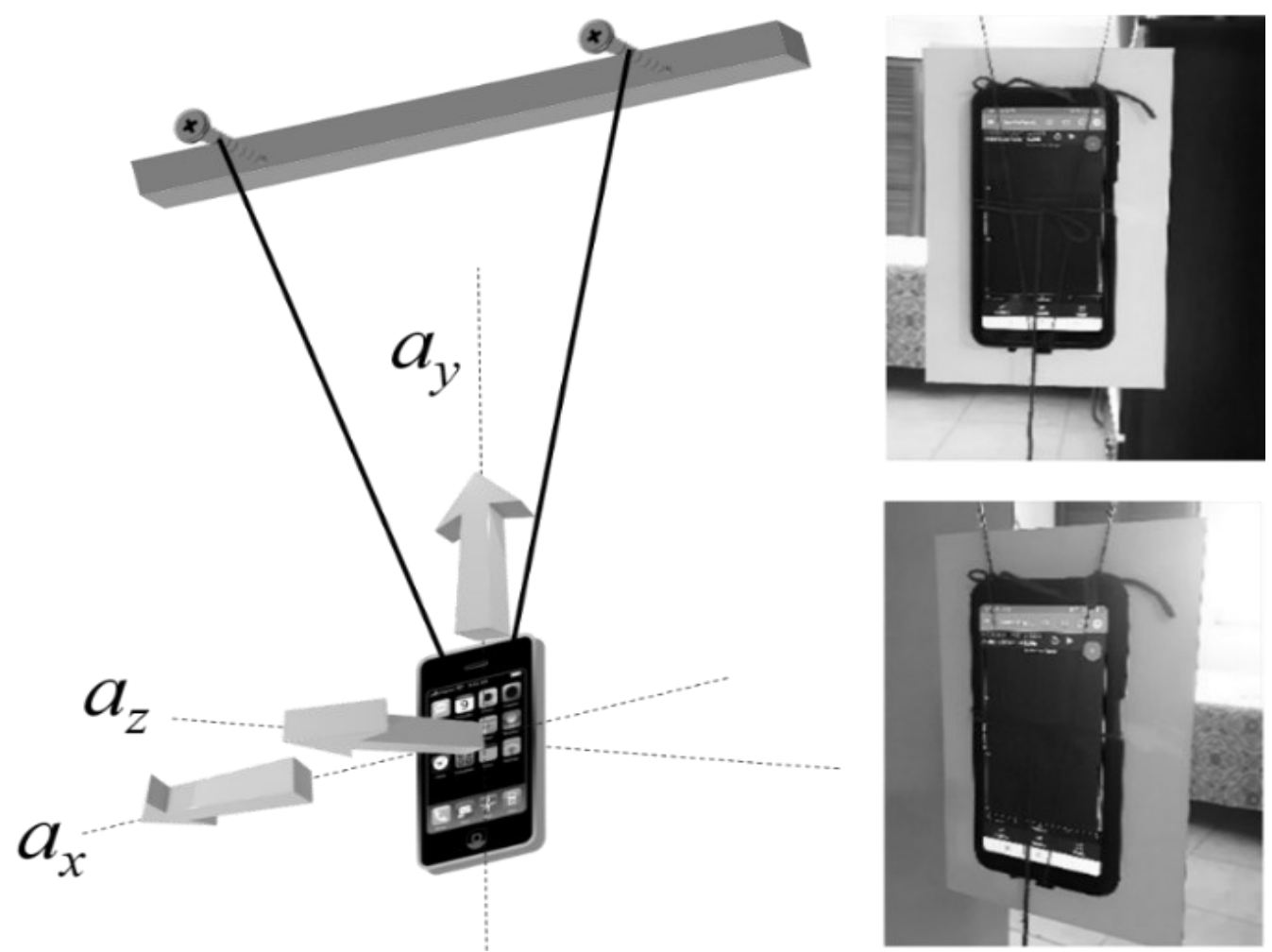

Fig. 2. (Left) An illustration of the oscillating pendulum body showing the acceleration components direction. (Right) Photos of the experimental setup.

The Physics Toolbox Sensor Suite application [16], freely available on Google Play for the Android platform, was used for data capture and storage. Stored data can be exported for analysis in other devices in .csv format.

\section{RESULTS AND DISCUSSION}

Fig. 3 shows the behavior of the time-dependent tangential and radial acceleration for each pendulum. It is possible to observe the periodic motion as the tangential acceleration changes direction, indicated by the positive and negative values of the vertical axis of the graphs, every half period of movement. The pendulum body remains under the action of the restoring force (net tangential force) oriented to the equilibrium position of the system. The time-dependent radial acceleration is characterized by presenting only positive values, as the net radial force always points towards the center of the circumference arc. 

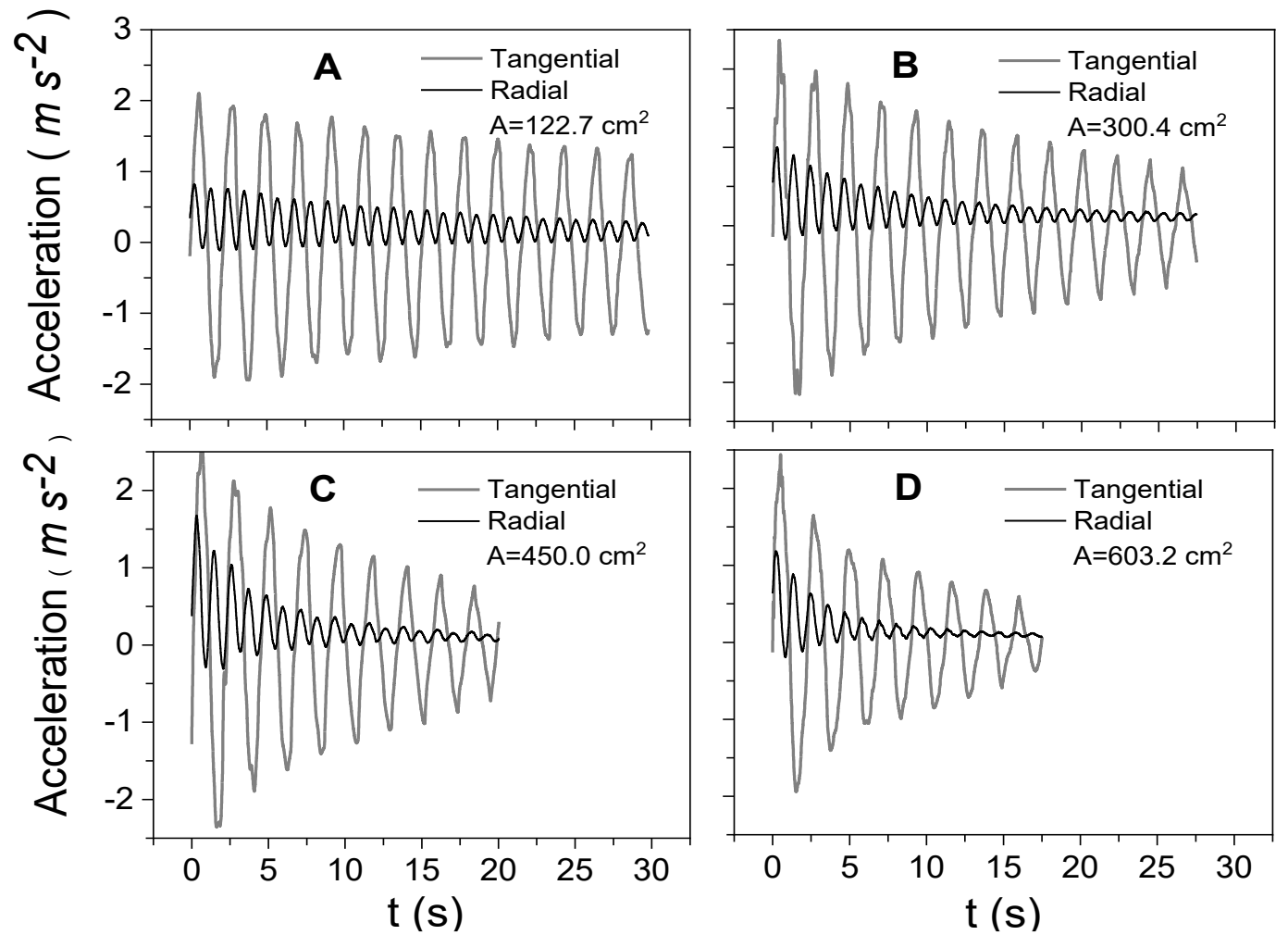

Fig. 3. Time-dependent tangential and radial acceleration under different damping conditions.

There is an evident decrease in oscillation amplitude with time for both accelerations. This decrease is more pronounced as the cross-section area is increased, a clear demonstration of the effect of the air drag.

Despite the remarkable decrease in the oscillation amplitude with time shown in fig. 3, no marked effect of the dissipative force (due to air drag) on the pendulum period is observed. For any specific time interval, the pendulum performs the same number of oscillations per second. An increase in the period is expected when air drag effect is significant.

Fig. 4 shows the linear behavior of the tangential acceleration oscillation amplitude $\left(a_{t-a m p}\right)$ with time on a semi-logarithmic scale, and a fitting of an exponential function to the data points. This simple exponential mathematical model was used to obtain the damping coefficient $(\gamma)$ and the angular frequency for each different cross-section area pendulum, presented in table 1. As expected, the damping coefficient increases with cross-section area. 


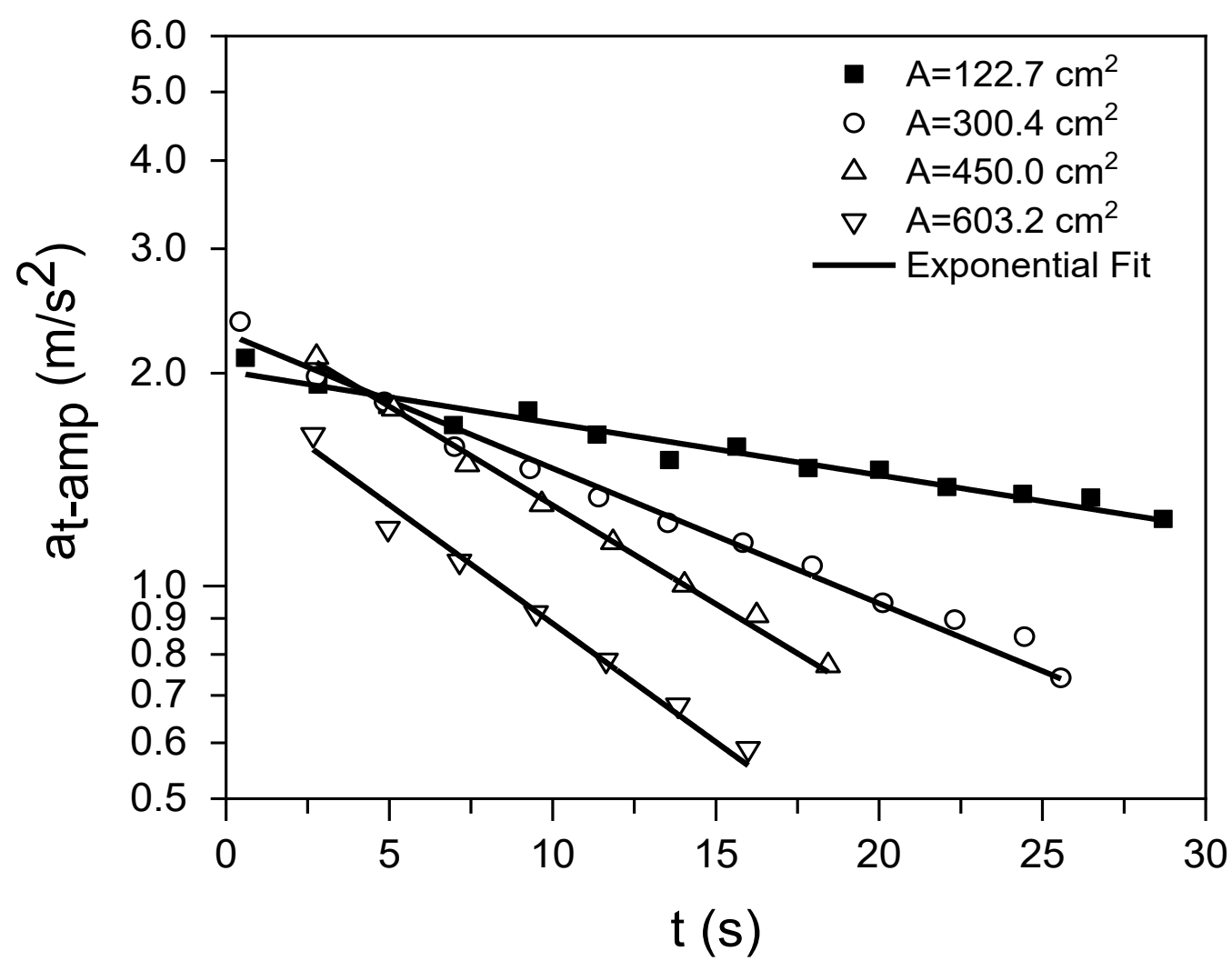

Fig. 4. Time-dependent tangential acceleration amplitude behavior for different damping conditions on a semi- logarithmic scale. For an increase in the area of the air barrier, an increase in the damping coefficient occurs. See slope of the graph.

Table 1 presents the values obtained from the analysis of the results shown in Fig. 3 and 4 and using the pendulum length in equation 7 to calculate the angular frequency.

TABLE I

Parameters extracted from fig. 3 and 4 for tangential acceleration. (A) Cardboard Air Barrier Area. Mathematical Model. ( $\gamma$ ) Damped Coefficient. (T) Average Oscillation Period. $\left(\mathrm{w}^{\prime}\right)$ Angular Frequency of Oscillation. The length of the pendulum is $116,5 \mathrm{~cm}$

\begin{tabular}{|c|c|c|c|c|}
\hline $\begin{array}{l}\text { Area } \\
\left(\mathrm{cm}^{2}\right)\end{array}$ & $\begin{array}{l}\text { Mathematical } \\
\text { model }\end{array}$ & $\gamma(1 / s)$ & $\mathrm{T}^{\prime}(\mathrm{s})$ & $\begin{array}{c}w^{\prime}=\sqrt{\frac{g}{l}-\gamma^{2}} \\
(\mathrm{rad} / \mathrm{s})\end{array}$ \\
\hline 122,7 & $k e^{-0,017 t}$ & $0,017 \pm 0,001$ & $2,162 \pm 0,089$ & $2,90 \pm 0,45$ \\
\hline 300,4 & $k e^{-0,044 t}$ & $0,044 \pm 0,002$ & $2,178 \pm 0,089$ & $2,90 \pm 0,46$ \\
\hline 450,0 & $k e^{-0,064 t}$ & $0,064 \pm 0,002$ & $2,206 \pm 0,047$ & $2,90 \pm 0,16$ \\
\hline 603,2 & $k e^{-0,077 t}$ & $0,077 \pm 0,006$ & $2,220 \pm 0,083$ & $2,89 \pm 0,46$ \\
\hline
\end{tabular}

The average period of oscillations slightly increases with cross-section area. But no significant effect of the dissipative force on the pendulum period is observed, despite the remarkable decrease in the oscillation amplitude with time.

No significant decrease in the angular frequency of the oscillations (Table 1) was observed, due to the low values of the damped coefficient, contributing less than thousandths of rad/s. Likewise, the average period of oscillation presents small differences from trial to trial of only fractions of a second. This result shows that under these experimental conditions the viscosity of the air does affect the amplitude of oscillations of the pendulum (Fig.3), but it does not affect its period (nor its frequency).

The effect of the cross-section area on the time needed to observe a reduction in the initial amplitude of the movement by half, called the half-life of the amplitude of oscillation ( $\left.\mathrm{t}_{1 / 2}\right)$ [17], was studied. Fig. 5 shows $\mathrm{t}_{1 / 2}$ versus cross-section area, and a power function fit, and it is observed that the time required to reduce the amplitude of oscillation by half is inversely proportional to the cross-section area of the pendulum. 


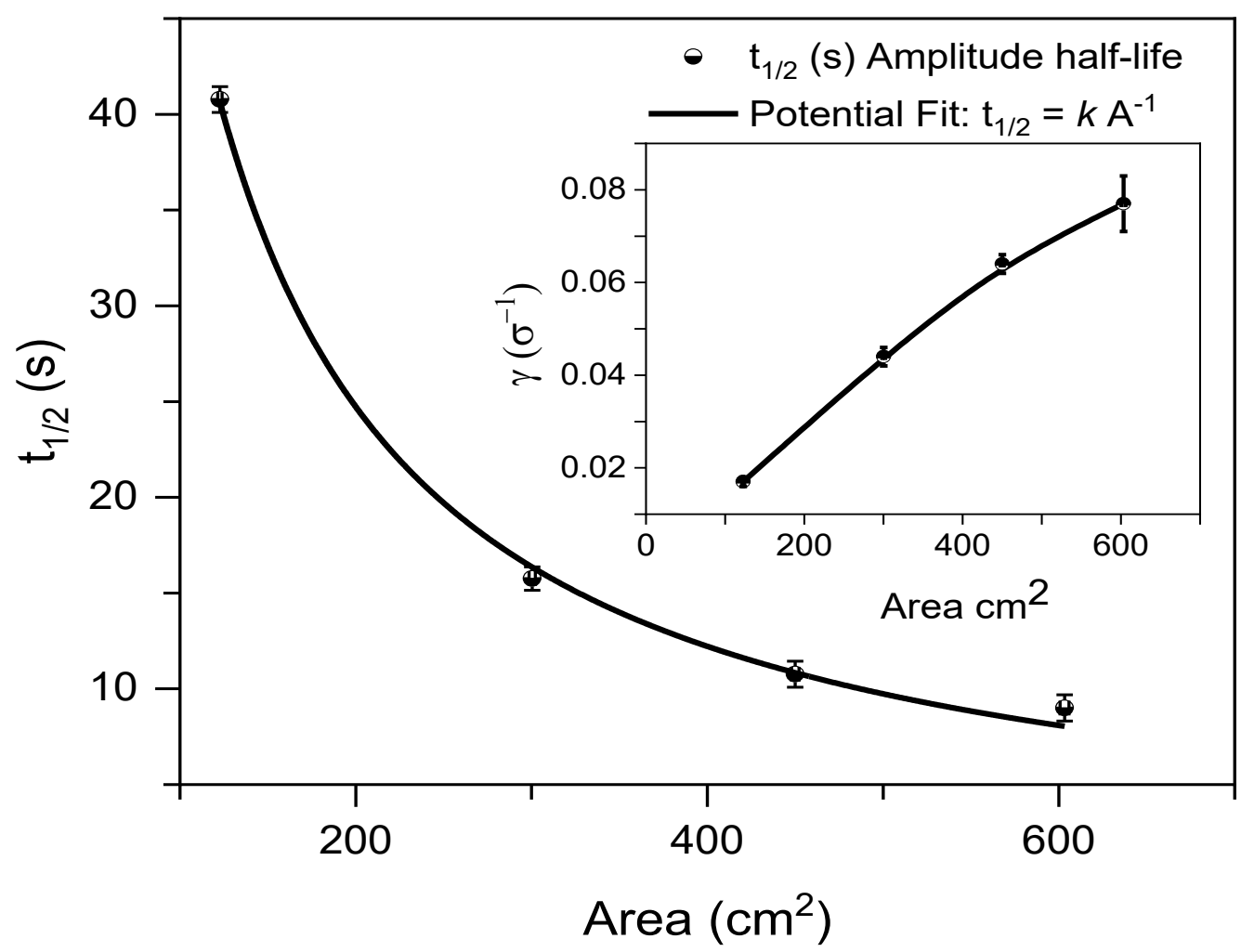

Fig. 5. Effect of the cross-section area of the pendulum on the half-life of the amplitude of oscillation. Inset: damping coefficient versus cross-section area of the pendulum.

\section{CONCLUSION}

The acceleration sensor of a Samsung Galaxy SM-A750G smartphone and the Physics Toolbox Sensor Suite app were used to investigate influence of the cross-section area on the behavior of an oscillating pendulum. Results show that the increment in the cross-section area from 123 to $603 \mathrm{~cm}^{2}$ slightly increased the damping coefficient from 0,017 to $0,077 \mathrm{~s}^{-1}$, and led to a significant decrease in the amplitude of oscillations with time.

On the other hand, no significant variation in the oscillation period was evident, as the observed variation was less than $60 \mathrm{~ms}$ when the cross-section area was varied. Likewise, no significant variation in the angular frequency of the oscillations was observed. So, air viscosity did not significantly affect the retarding force acting on the system.

The dependence of the amplitude half-life on the cross-section area was modeled using a power function, and the model showed that the time required to reduce the amplitude of the oscillations by half is inversely proportional to the cross-section area of the pendulum.

The results showed that smartphones are effective and reliable instruments to be used in academic activities, making it possible to present students with a motivating way for learning new physical concepts. As a popular technology, available to most students, it offers the opportunity to carry out experiments both in the classroom, in the laboratory, or at home.

\section{REFERENCES}

[1] Google, "Android Open-Source Project," May 04, 2020. [Online]. Available: https://source.android.com/devices/sensors/sensor-types. [Accessed July 06, 2020].

[2] M. Susi, D. Borio and D. Lachapelle, «Accelerometer Signal Features and Classification Algorithms for Positioning Applications, » de International Technical Meeting of The Institute of Navigation 2011, Session A1, San Diego, CA, 2011.

[3] Google, "Google Play," 2020. [Online]. Available: https://play.google.com/store/apps/details?id=imoblife.androidsensorbox\&hl=es_PA. [Accessed July 17, 2020].

[4] Google Play, Google, 2020. [Online]. Available: https://play.google.com/store/apps/details?id=com.innoventions.sensorkinetics\&hl=es_PA. [Accessed July 17, 2020].
A.
University,
"PhyPhox
Physical
Phone
Experiments,"2020.

[Online].

Available:

[6] Google, "Science Journal," 2020. [Online]. Available: https://sciencejournal.withgoogle.com/. [Accessed August 2, 2020].

[7] P. Vogt and J. Kuhn, "Analyzing free fall with a smartphone acceleration sensor," The Physics Teachers, vol. 50, no. 182, pp. 182 - 183, 2012.

[8] P. Vogt and J. Kuhn, "Analyzing simple pendulum phenomena with a smartphone acceleration sensor," The Physics Teacher, vol. 50, no. 439, pp. 439 - 440, 2012.

[9] A. Shakur and J. Kraft, "Measurement of Coriolis Acceleration with a Smartphone," The Physics Teacher, vol. 54, no. 288 , pp. 288 - $290,2016$.

[10] J. R. Groff, « Estimating RC Time Constants Using Sound, "The Physics Teacher, vol. 57, nº 393, pp. 393 - 396, 2019. 
[11] C. Medina, S. Velazco and J. Salinas, "Control Experimental del Modelo de Péndulo Matemático," Revista Brasileira de Ensino de Física, vol. 24, no. 2, pp. $254-258,2002$.

[12] M. Alonso and E. J. Finn, Física, Vol. 1. Mechanics, First Edition in Spanish, 1970, p. 387.

[13] R. A. Serway and J. W. Jewett, Física para Ciencias e Ingeniería, 9 ed., Vol. 1, México D. F.: Cengage Learning, 2015 , pp. 464.

[14] R. A. Serway and J. W. Jewett, Física para Ciencia e Ingeniería, 9 ed., Vol. 1. México D. F.: Cengage Learning, 2015, pp. 162.

[15] MovilCelular, «CC MovilCelular,» [Online]. Available: https://www.movilcelular.es/especificaciones/samsung/galaxy-a7-2018/sm-a750gds-128gb/. [Last access: June 18, 2010].

[16] V. Software, "Physics Toolbox Apps," [Online]. Available: https://www.vieyrasoftware.net/. [Accessed June 18, 2020].

[17] R. D. Knight, Physics for Scientists and Engineers, Fourth Edition ed., Boston: Pearson Education, 2017, pp. 410. 